\title{
A Study of Two Dimensional Unsteady MHD Free Convection Flow over a Vertical Plate in the Presence of Radiation
}

\author{
Mohammad Sharif Ullah $1,2^{*}$ (D , Absana Tarammim²,3, Mohammed Jahir Uddin ${ }^{3,4}$ \\ ${ }^{1}$ Department of Mathematics, Feni University, Feni, Bangladesh \\ ${ }^{2}$ Department of Mathematics, Bangladesh University of Engineering and Technology (BUET), Dhaka, Bangladesh \\ ${ }^{3}$ Department of Mathematics, Chittagong University of Engineering and Technology (CUET), Chittagong, Bangladesh \\ ${ }^{4}$ Department of Mathematics, Feni Government College, Feni, Bangladesh \\ Email: *sharifju49@yahoo.com, *sharifjnu561@gmail.com
}

How to cite this paper: Ullah, M.S., Tarammim, A. and Uddin, M.J. (2021) A Study of Two Dimensional Unsteady MHD Free Convection Flow over a Vertical Plate in the Presence of Radiation. Open Journal of Fluid Dynamics, 11, 20-33. https://doi.org/10.4236/ojfd.2021.111002

Received: October 4, 2020

Accepted: January 29, 2021

Published: February 2, 2021

Copyright $\odot 2021$ by author(s) and Scientific Research Publishing Inc. This work is licensed under the Creative Commons Attribution International License (CC BY 4.0).

http://creativecommons.org/licenses/by/4.0/

\begin{abstract}
This paper compacts with an exact analysis of radiative effects on the magnetohydrodynamic (MHD) free convection flow of an electrically conducting incompressible viscous fluid over a vertical plate. The non-dimensional continuity, momentum, and energy equations are solved using appropriate transformation. The dimensionless momentum and energy equations are solved numerically through an explicit finite difference method. The stability and convergence analysis also discussed. Finally, outcomes of the parameters on velocity and temperature profiles are displayed graphically and qualitatively.
\end{abstract}

\section{Keywords}

Heat Transfer, Mass Transfer, MHD, Vertical Plate, Convection Flow

\section{Introduction}

Due to the plethora of applications in astronomical technology and processes entangling high temperatures, the payoffs of thermal radiation on the free convection flows have been drawing the consideration of enormous research interest. Furthermore, free convection flow in the presence of a magnetic field is crucial because of its significant impact on the boundary layer control and the execution of many engineering devices consuming electrically conducting fluids such as in MHD power generation, plasma studies, nuclear reactor using a liquid metal coolant and geothermal energy extraction. That's why it's a burning topic for many current researchers in the world. 
With the consideration of radiation impacts, the energy equation has to lead to an exceptionally nonlinear partial differential equation. In 1960, Soundalgekar et al. [1] scrutinized the radiation effects on free convection flow of gas past a semi-infinite flat plate. In 1996, Hossain et al. [2] investigated the radiation effect on mixed convection along with a vertical plate and uniform surface temperature. In 1996, Das et al. [3] evaluated the radiation outcome of flow past an impulsively started infinite isothermal vertical plate, using the Laplace transform technique. In 1999, Hossain et al. [4] investigated the offshoot of radiation on free convection from a porous vertical plate. In 1999, Raptis and Perdikis [5] examined the upshot of thermal radiation and free convection flow past a moving plate. In 2001, Hossain and Pop [6] investigated the radiation effect on free convection over a vertical flat plate embedded in a porous medium with high porosity. In 2001, Chamkha [7] investigated the coupled heat and mass transfer by natural convection of Newtonian fluids in the presence of magnetic field and radiation effects. In 2001, Hossain et al. [8] studied the impact of radiation on free convection flow of fluid with variable viscosity from a porous vertical plate. In 2003, Cookey et al. [9] well-acquainted the influence of viscous dissipation and radiation on unsteady MHD free-convection flow past an infinite heated vertical plate in a porous medium with time-dependent suction. In 2003, Chamkha et al. [10] resolved the thermal radiation effects on MHD forced convection flow adjacent to a non-isothermal wedge in the presence of a heat source or sink. In 2004, Abd EL-Naby et al. [11] calculated the finite difference solution of radiation effects on MHD unsteady free-convection flow on a vertical porous plate. In 2005, Makinde [12] studied the free-convection flow with thermal radiation and mass transfer past a moving vertical porous plate. In 2005 and 2006, Duwairi [13] and Damseh et al. [14] examined the radiation and magnetic effects on the skin friction and heat transfer for forced convection conditions. In 2008, Aydın and Kaya [15] observed the payoffs of thermal radiation taking into account the suction or injection on a steady MHD mixed convective flows of a viscous incompressible fluid about a permeable vertical plate. In 2008, Cortell [16] presented a numerical analysis for flow and heat transfer in a viscous fluid over a sheet nonlinearly stretched with effects of thermal radiation. In 2009, Deka and Neog [17] [18] reviewed the combined payoffs of thermal radiation and chemical reaction on free convection flow past a vertical plate in a porous medium and with MHD. In 2010, Roy and Hossain [19] traced the natural convection boundary layer flow of a viscous incompressible fluid along a vertical plate taking into account the conduction-radiation interaction and surface temperature oscillations. In 2011, Seth et al. [20] investigated the impacts of radiation on unsteady hydromagnetic natural convection transient flow near an impulsively moving vertical flat plate with ramped wall temperature in a porous medium. In 2012, Das [21] mastered the yielding of thermal radiation on a steady two-dimensional boundary layer flow of an incompressible electrically conducting fluid over a flat plate assuming the partial slip at the surface of the boundary, temperature-dependent fluid viscosity, variable thermal conductivity, 
and non-uniform heat generation and absorption. In 2012, Khan et al. [22] quested the unsteady MHD free convection boundary-layer flow of a nano-fluid along a stretching sheet with thermal radiation and viscous dissipation effects. In 2012, Rao et al. [23] studied the radiation corporeity of unsteady free convection heat and mass transfer in a walters-B viscoelastic flow past an impulsively started vertical plate. In 2014, Roy et al. [24] superintendence the unsteady MHD free convection flow along with a vertical plate in the presence of radiative heat flux. In 2014, Samad and Saha [25] examined the two-dimensional heat and mass transfer free convection flow of an MHD Non-Newtonian power-law fluid along with a stretching sheet in the presence of a magnetic field and assuming simplified thermal radiation. In 2014, Fetecau et al. [26] smeared the slip effects on the unsteady radiative MHD free convection flow over a moving plate with mass diffusion and heat source. In 2015, Nagamanemma et al. [27] analyzed unsteady MHD free convective heat and mass transfer flow near a moving vertical porous plate with radiation and thermo diffusion effects. In 2015, Tomer and Kumar [28] perused the radiation effects on heat and mass transfer in a steady MHD flow over a porous vertical plate. In 2020, Tarammim et al. [29] studied two dimensional unsteady MHD free convection flow over a vertical plate.

The literature review discloses that many researchers work on steady heat transfer due to a variety of physical parameters. Although some research works are considered the unsteady problem used as simplified models, disregarding the results of the appearance of the magnetic field or thermal radiation. Analyzing this paper, we scrutinize the unsteady MHD free convection flow over a vertical plate in the presence of thermal radiation. We solve the governing equations with an explicit finite difference method. Then the upshot of the physical parameters such as the radiative parameter $R$, magnetic parameter $M$, Prandtl number $P r$, Grashof number $G r$, and Ekert number $E c$ are presented here.

\section{Mathematical Model and Governing Equations}

Let us consider unsteady MHD free convection flow with electrically conducting incompressible viscous fluid along a vertical plate in presence of radiation. The Cartesian coordinate system, the $\mathrm{X}$-axis is taken along the plate in the upward direction and the Y-axis is normal to plate. $T_{w}$ is the temperature of the plate and $T_{\infty}$ outside of the plate separately. A uniform magnetic field $\boldsymbol{B}=\left(0, B_{0}, 0\right)$ is enacted normal to the plate, and the magnetic field is anticipated to be negligible while $B_{0}$ is constant, which demonstrates in Figure 1.

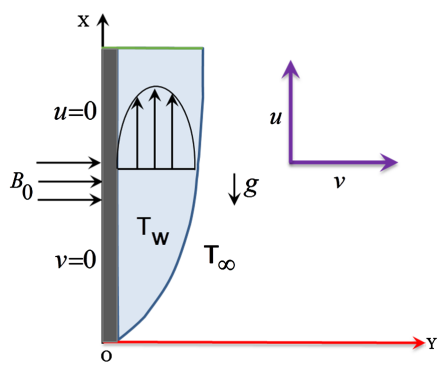

Figure 1. Physical configuration and coordinates system. 
The equations for unsteady MHD heat transfer flow over a vertical plate in the presence of radiation with boundary conditions are given below:

Continuity equation

$$
\frac{\partial u}{\partial x}+\frac{\partial v}{\partial y}=0
$$

Momentum equation

$$
\frac{\partial u}{\partial t}+u \frac{\partial u}{\partial x}+v \frac{\partial u}{\partial y}=v \frac{\partial^{2} u}{\partial y^{2}}+g \beta\left(T-T_{\infty}\right)-\frac{\sigma B_{0}^{2}}{\rho} u
$$

Energy equation

$$
\frac{\partial T}{\partial t}+u \frac{\partial T}{\partial x}+v \frac{\partial T}{\partial y}=\frac{\kappa}{\rho C_{p}} \frac{\partial^{2} T}{\partial y^{2}}+\frac{v}{C_{p}}\left(\frac{\partial u}{\partial y}\right)^{2}-\frac{1}{\rho C_{p}} \frac{\partial q_{r}}{\partial y}
$$

The relevant boundary condition for velocity and temperature are given by

$$
\begin{aligned}
& u=U_{0}, v=0, T=T_{w}, \text { at } y=0 \\
& u \rightarrow 0, T \rightarrow T_{\infty}, \text { as } y \rightarrow \infty
\end{aligned}
$$

where $\beta$ is the co-efficient of volumetric expansion, $v$ is the kinematic viscosity, $g$ is the acceleration due to gravity, $T$ is the temperature of the fluid inside the thermal boundary layer, $T_{w}$ is the temperature of the plate, $T_{\infty}$ is the temperature in the free stream, $\sigma$ is the electric conductivity, $B_{0}$ is a constant magnetic field, $\rho$ is the fluid density, $\kappa$ is the kinematic viscosity, $C_{p}$ is the specific heat with constant pressure, $U_{0}$ is a constant indicates the uniform velocity of the fluid remaining symbols have their usual meaning.

Conferring to Rosseland approximation the radiative heat flux, $q_{r}=-\frac{4 \sigma^{*}}{3 \kappa_{0}} \frac{\partial T^{4}}{\partial y}$, where $\kappa_{0}$ is the Rosseland mean absorption coefficient and $\sigma^{*}$ is the Stefan-Boltzman constant.

The Rosseland approximation is intended for an optically thick medium, so the fluid does not absorb its particular emitted radiation, but it does absorb radiation emitted by the boundaries without self-absorption, It seems reasonable to assume that the temperature differences within the flow are expected to be small and $T^{4}$ can be articulated as a linear function of the temperature. Thus $T^{4}$ can be extended in Taylor series about $T_{\infty}$ as:

$$
T^{4}=T_{\infty}^{4}+4 T_{\infty}^{3}\left(T-T_{\infty}\right)+6 T_{\infty}^{2}\left(T-T_{\infty}\right)^{2}+\cdots,
$$

Neglecting the higher order terms elsewhere the first degree in $\left(T-T_{\infty}\right)$ one can be found as $T^{4} \approx-3 T_{\infty}^{4}+4 T_{\infty}^{3} T$.

$$
\therefore q_{r}=-\frac{16 \sigma^{*}}{3 \kappa_{0}} T_{\infty}^{3} \frac{\partial T}{\partial Y} \text {. }
$$

By replacing the above expression into energy Equation (3) are as follows:

$$
\frac{\partial T}{\partial t}+u \frac{\partial T}{\partial x}+v \frac{\partial T}{\partial y}=\frac{\kappa}{\rho C_{p}} \frac{\partial^{2} T}{\partial y^{2}}+\frac{v}{C_{p}}\left(\frac{\partial u}{\partial y}\right)^{2}+\frac{1}{\rho C_{p}} \frac{16 \sigma^{*}}{3 \kappa_{0}} T_{\infty}^{3} \frac{\partial^{2} T}{\partial y^{2}}
$$




\section{Mathematical Formulation}

Applying the ensuing usual transformations, the system of partial differential equations with boundary conditions is changed into a dimensionless equation.

$$
u=U_{0} U, v=V U_{0}, Y=\frac{y U_{0}}{v}, X=\frac{x U_{0}}{v}, \eta=\frac{t U_{0}^{2}}{v}, T=T_{\infty}+\left(T_{w}-T_{\infty}\right) \bar{T}
$$

Applying the above transformation in Equations (1), (2), (3), and with corresponding boundary conditions (4), after simplification we acquire the following non-linear differential equations in terms of dimensionless variables such as:

Continuity equation

$$
\frac{\partial U}{\partial X}+\frac{\partial V}{\partial Y}=0
$$

Momentum equation

$$
\frac{\partial U}{\partial \eta}+U \frac{\partial U}{\partial X}+V \frac{\partial U}{\partial Y}=\frac{\partial^{2} U}{\partial Y^{2}}+G r \bar{T}-M U
$$

Energy equation

$$
\frac{\partial \bar{T}}{\partial \eta}+U \frac{\partial \bar{T}}{\partial X}+V \frac{\partial \bar{T}}{\partial Y}=\frac{1}{\operatorname{Pr}} \frac{\partial^{2} \bar{T}}{\partial Y^{2}}+E c\left(\frac{\partial U}{\partial Y}\right)^{2}+R \frac{\partial^{2} \bar{T}}{\partial Y^{2}}
$$

with boundary conditions

$$
\begin{aligned}
& U=1, V=0, \bar{T}=1, \text { at } Y=0 \\
& U=0, \bar{T}=0, \text { as } Y \rightarrow \infty
\end{aligned}
$$

where,

Magnetic parameter, $M=\frac{\sigma v B_{0}^{2}}{\rho U_{0}^{2}}$

Grashof number, $G r=\frac{\nu g \beta\left(T_{w}-T_{\infty}\right)}{U_{0}^{3}}$

Prandtl number, $\operatorname{Pr}=\frac{v}{\alpha}$

Eckert number, $E c=\frac{U_{0}^{2}}{C_{p}\left(T_{w}-T_{\infty}\right)}$

Radiative parameter, $R=\frac{16 \sigma^{*} T_{\infty}^{3}}{3 \rho C_{p} \kappa_{0}}$.

\section{Numerical Solution}

A set of nonlinear partial differential dimensionless governing equations has been brought out numerically with the related boundary conditions along with an explicit finite difference method, which is tentatively stable. The portion of the flow is divided into a grid or mesh of lines parallel to $X$ - and $Y$-axes, where $X$-axis indicates the plate in upward direction and $Y$-axis is normal to the plate. We measure the height of plate $X_{\max } \quad(=100)$, i.e., $X$ differs from 0 to 100 and suppose $Y_{\max } \quad(=25)$ as taken to $Y \rightarrow \infty$, it means that $Y$ varies from 0 to 25 . 
Let, $m=250$ and $n=250$ grid spacing in $X$ and $Y$ directions correspondingly and as follows $\Delta x=0.4(0 \leq x \leq 100)$ and $\Delta Y=0.1(0 \leq Y \leq 25)$ with the minor time period $\Delta \eta=0.005$. Let $U^{\prime}, \overline{T^{\prime}}$ indicate the values of $U, \bar{T}$ at the terminal of a time-step separately.

Applying an explicit finite difference method into the partial Equations (6)-(8) with boundary conditions (9) we get,

$$
\begin{gathered}
(6) \Rightarrow \frac{U_{i, j}-U_{i, j-1}}{\Delta X}+\frac{V_{i, j}-V_{i, j-1}}{\Delta Y}=0 \\
(7) \Rightarrow \frac{U_{i, j}^{\prime}-U_{i, j}}{\Delta \eta}+U_{i, j} \frac{U_{i, j}-U_{i-1, j}}{\Delta X}+V_{i, j} \frac{U_{i, j+1}-U_{i, j}}{\Delta Y} \\
=\frac{U_{i, j+1}-2 U_{i, j}+U_{i, j-1}}{(\Delta Y)^{2}+G r \bar{T}_{i, j}-M U_{i, j}} \\
\Rightarrow U_{i, j}^{\prime}=U_{i, j}+\Delta \eta\left(-U_{i, j} \frac{U_{i, j}-U_{i-1, j}}{\Delta X}-V_{i, j} \frac{U_{i, j+1}-U_{i, j}}{\Delta Y}\right. \\
(8) \Rightarrow \frac{\bar{T}_{i, j}^{\prime}-\bar{T}_{i, j}}{\Delta \eta}+U_{i, j} \frac{\bar{T}_{i, j}-\bar{T}_{i-1, j}}{\Delta X}+V_{i, j} \frac{\bar{T}_{i, j+1}-\bar{T}_{i, j}}{\Delta Y} \\
=\frac{1}{P r} \frac{\bar{T}_{i, j+1}-2 \bar{T}_{i, j}+\bar{T}_{i, j-1}+E c\left(\frac{U_{i, j+1}-U_{i, j}}{\Delta Y}\right)^{2}+R \frac{\bar{T}_{i, j+1}-2 \bar{T}_{i, j}+\bar{T}_{i, j-1}}{(\Delta Y)^{2}}}{(\Delta Y)^{2}} \\
\Rightarrow \bar{T}_{i, j}^{\prime}=\bar{T}_{i, j}+\Delta \eta\left(-U_{I, J} \frac{\bar{T}_{i, j}-\bar{T}_{i-1, j}}{\Delta X}-V_{i, j} \frac{\bar{T}_{i, j+1}-\bar{T}_{i, j}}{\Delta Y}\right. \\
+\frac{1}{P r} \frac{\bar{T}_{i, j+1}-2 \bar{T}_{i, j}+\bar{T}_{i, j-1}}{(\Delta Y)^{2}}+E c\left(\frac{U_{i, j+1}-U_{i, j}}{\Delta Y}\right)^{2} \\
\left.+R \frac{\bar{T}_{i, j+1}-2 \bar{T}_{i, j}+\bar{T}_{i, j-1}}{(\Delta Y)^{2}}\right)
\end{gathered}
$$

The boundary conditions with the finite difference methods are as follows:

$$
\begin{gathered}
U_{i, 0}^{n}=1, V_{i, 0}^{n}=0, \bar{T}_{i, 0}^{n}=1 \\
U_{i, L}^{n}=0, \bar{T}_{i, L}^{n}=0,
\end{gathered}
$$

where

$$
L \rightarrow \infty
$$

where, $i$ and $j$ indicate the grid points with $X$ and $Y$ coordinates correspondingly and the subscripts $\mathrm{n}$ represents a value of time. $\bar{T}$ is the temperature.

\section{Stability and Convergence Analysis}

Analysis will keep on inadequate without the stability and convergence analysis. It's always supportive of tangible computations. Furthermore, it establishes numerical solutions (finite difference scheme) that are reliable and consistent. Owing to the persistent of mesh sizes, the stability criteria of the problem is 
formulated as:

The Fourier expansion due to $U, \theta, \varphi$ at time arbitrary say $\eta=0$ is $\mathrm{e}^{i \bar{\alpha} \bar{x}}$ and $\mathrm{e}^{i \bar{\beta} \bar{y}}$ apart from a constant, where $i=\sqrt{-1}$.

Then

$$
\begin{aligned}
& U: \Psi(\eta) \mathrm{e}^{i \bar{\alpha} \bar{x}} \mathrm{e}^{i \bar{\beta} \bar{y}} \\
& \bar{T}: \theta(\eta) \mathrm{e}^{i \bar{\alpha} \bar{x}} \mathrm{e}^{i \bar{\beta} \bar{y}}
\end{aligned}
$$

Subsequently the time period, Equation (14) will convert

$$
\begin{aligned}
& U: \Psi^{\prime}(\eta) \mathrm{e}^{i \bar{\alpha} \bar{x}} \mathrm{e}^{i \bar{\beta} \bar{y}} \\
& \bar{T}: \theta^{\prime}(\eta) \mathrm{e}^{i \bar{\alpha} \bar{x}} \mathrm{e}^{i \bar{\beta} \bar{y}}
\end{aligned}
$$

Applying Equations (14) and (15) into Equations (11) and (12), the following equations we found by simplification.

$$
\begin{aligned}
& \frac{\Psi^{\prime}-\Psi}{\Delta \eta}+U \frac{\Psi\left(1-\mathrm{e}^{-i \bar{\alpha} \Delta X}\right)}{\Delta X}+V \frac{\Psi\left(\mathrm{e}^{i \bar{\beta} \Delta Y}-1\right)}{\Delta Y} \\
& =\frac{2 \Psi(\Delta Y \cos \beta-1)}{(\Delta Y)^{2}}+G r \theta^{\prime}-M \Psi \\
& \Rightarrow \Psi^{\prime}-\Psi+\frac{\Delta \eta}{\Delta X} U \Psi\left(1-\mathrm{e}^{-i \bar{\alpha} \Delta X}\right)+\frac{\Delta \eta}{\Delta Y} V \Psi\left(\mathrm{e}^{i \bar{\beta} \Delta Y}-1\right) \\
& =2 \frac{\Delta \eta}{(\Delta Y)^{2}} \Psi(\Delta Y \cos \beta-1)+G r \Delta \eta \theta^{\prime}-M \Psi \\
& \Rightarrow \Psi^{\prime}=\Psi\left\{1-\frac{\Delta \eta}{\Delta X} U\left(1-\mathrm{e}^{-\bar{\alpha} i \Delta X}\right)-\frac{\Delta \eta}{\Delta Y} V\left(\mathrm{e}^{i \bar{\beta} \Delta Y}-1\right)\right. \\
& \left.+\frac{2 \Delta \eta}{(\Delta Y)^{2}}(\Delta Y \cos \beta-1)-M \Delta \eta\right\}+G r \Delta \eta \theta^{\prime}
\end{aligned}
$$

where

$$
\begin{gathered}
A=1-\frac{\Delta \eta}{\Delta x} U\left(1-\mathrm{e}^{-i \bar{\alpha} \Delta X}\right)-\frac{\Delta \eta}{\Delta Y} V\left(\mathrm{e}^{i \bar{\beta} \Delta Y}-1\right)+\frac{2 \Delta \eta}{(\Delta Y)^{2}}(\cos \beta \Delta Y-1)-M \Delta \eta \\
B=G r \Delta \eta
\end{gathered}
$$

and

$$
\begin{gathered}
\frac{\theta^{\prime}(\eta)-\theta(\eta)}{\Delta \eta}+U \theta(\eta) \frac{1-\mathrm{e}^{-i \bar{\alpha} \Delta X}}{\Delta X}+V \theta(\eta) \frac{\mathrm{e}^{-i \bar{\beta} \Delta Y}-1}{\Delta Y} \\
=\frac{1}{P r} \frac{2 \theta(\eta)(\cos \beta \Delta Y-1)}{(\Delta Y)^{2}}+E c\left(\frac{U \Psi(\eta)\left(\mathrm{e}^{i \bar{\beta} \Delta Y}-1\right)}{(\Delta Y)^{2}}\right)+R \frac{2 \theta(\eta)(\cos \beta \Delta Y-1)}{(\Delta Y)^{2}} \\
\Rightarrow \theta^{\prime}(\eta)=\theta(\eta)\left\{1-\frac{\Delta \eta}{\Delta x} U\left(1-\mathrm{e}^{-i \bar{\alpha} \Delta X}\right)-\frac{\Delta \eta}{\Delta Y} V\left(\mathrm{e}^{i \bar{\beta} \Delta Y}-1\right)\right. \\
\left.+\frac{1}{\operatorname{Pr}} \frac{2 \Delta \eta}{(\Delta Y)^{2}}(\cos \beta \Delta Y-1)+R \frac{2(\cos \beta \Delta Y-1)}{(\Delta Y)^{2}}\right\} \\
+E c U \frac{\Delta \eta}{(\Delta Y)^{2}} \Psi(\eta)\left(\mathrm{e}^{i \bar{\beta} \Delta Y}-1\right)
\end{gathered}
$$




$$
\Rightarrow \theta^{\prime}(\eta)=G \theta+H \Psi
$$

where,

$$
\begin{aligned}
G= & 1-\frac{\Delta \eta}{\Delta X} U\left(1-\mathrm{e}^{-i \bar{\alpha} \Delta X}\right)-\frac{\Delta \eta}{\Delta Y} V\left(\mathrm{e}^{i \bar{\beta} \Delta Y}-1\right) \\
& +\frac{1}{\operatorname{Pr}} \frac{2 \Delta \eta(\cos \beta \Delta Y-1)}{(\Delta Y)^{2}}+R \frac{2(\cos \beta \Delta Y-1)}{(\Delta Y)^{2}}
\end{aligned}
$$

and $H=E c U \frac{\Delta \eta}{(\Delta Y)^{2}}\left(\mathrm{e}^{i \overline{\beta \Delta} Y}-1\right)$.

Equations (16), (17) can be written as:

$$
\begin{aligned}
\Psi^{\prime} & =A \Psi+B(G \theta+H \Psi)=(A+H) \Psi+B G \theta \\
& \Rightarrow \Psi^{\prime}=A_{1} \Psi+B_{1} \theta
\end{aligned}
$$

where, $A_{1}=A+H$

$$
B_{1}=B G
$$

and

$$
\theta^{\prime}=G \theta+H \Psi
$$

Equations (18) and (19) can be expressed in matrix form.

$$
\begin{gathered}
\left(\begin{array}{l}
\psi^{\prime} \\
\theta^{\prime}
\end{array}\right)=\left(\begin{array}{ll}
A_{1} & B_{1} \\
H & G
\end{array}\right)\left(\begin{array}{l}
\psi \\
\theta
\end{array}\right) \\
\text { i.e. } \eta^{\prime}=T \eta
\end{gathered}
$$

where, $T=\left(\begin{array}{ll}A_{1} & B_{1} \\ H & G\end{array}\right)$ and $\eta=\left(\begin{array}{l}\psi \\ \theta\end{array}\right)$.

As eigenvalues of the augmentation matrix $T$ is crucial for attaining the stability condition, as a result, let

$$
B_{1} \rightarrow 0, \quad H \rightarrow 0 .
$$

Hence, matrix $T$ is as follows:

$$
T=\left(\begin{array}{cc}
A_{1} & 0 \\
0 & G
\end{array}\right)
$$

Thus, the Eigen values of $T$ are

$$
\lambda_{1}=A_{1}, \quad \lambda_{2}=G
$$

Here, values of $\lambda_{1}, \lambda_{2}$ must not surpass in modulus.

Therefore, the stability conditions are:

$$
\left|A_{1}\right| \leq 1, \quad|G| \leq 1 .
$$

Let, $a=\frac{u \Delta \eta}{\Delta X}, b=\frac{\Delta \eta}{\Delta Y} V, c=\frac{\Delta \eta}{(\Delta Y)^{2}}$.

Hence, $A=1-a\left(1-\mathrm{e}^{-i \bar{\alpha} \Delta X}\right)-b\left(\mathrm{e}^{i \bar{\beta} \Delta Y}-1\right)+2 c(\cos \beta \Delta Y-1)-M \Delta \eta$

$$
H=E c U c\left(C^{i \bar{\beta} \Delta Y}-1\right) \text {. }
$$


The co-efficient of $a, b, c$ are real and non-negative. Therefore, the maximum modulus of $A_{1}, G$ arise when $\bar{\alpha} \Delta X=m \pi$ and $\bar{\beta} \Delta Y=n \pi$ where $m$ and $n$ are integer and therefore $A_{1}, G$ are real. The values of $\left|A_{1}\right|,|G|$ are greatest when $\mathrm{m}$ and $\mathrm{n}$ are odd integers, then

$$
A_{1}=A+H=1-2 a-2 b-4 c-2 c E c .
$$

To satisfy $\left|A_{1}\right| \leq 1,|G| \leq 1$ the most negative permissible values are,

$$
A_{1}=-1, \quad G=-1 .
$$

Therefore, the stability conditions of the problem are assumed below.

$$
\begin{aligned}
& 1-2 a-2 b-4 c-2 c E c \leq-1 \\
& 2(a+b+2 c+c E c) \leq 2 \\
& a+b+2 c+c E c \leq 1 \\
& \frac{U \Delta \eta}{\Delta X}+\frac{|V| \Delta \eta}{\Delta Y}+\frac{2 \Delta \eta}{(\Delta Y)^{2}}+\frac{\Delta \eta}{(\Delta Y)^{2}} E c \leq 1
\end{aligned}
$$

Analogously,

$$
\frac{U \Delta \eta}{\Delta X}+\frac{|V| \Delta \eta}{\Delta Y}+\frac{1}{\operatorname{Pr}} \frac{2 \Delta \eta}{(\Delta Y)^{2}}
$$

and convergence criteria of the method is $\operatorname{Pr} \geq 1$.

\section{Results and Discussion}

For rummaging the problem, the outcome of numerical values of dimensionless velocity and temperature profiles within the boundary conditions have been enumerated due to several values of radiation parameter $R$, magnetic parameter $M$, Prandtl number $P r$, Grashof number $G r$, Eckert number $E c$ respectively. Furthermore, computed results of velocity and temperature profiles are portrayed and physical explanation explained here.

\subsection{Effect of Radiative Parameter $(R)$}

Figure 2(a-i) indicates that the velocity profile declines for ascending values of $R$. Whereas the boundary layer thickness falls down owing to rising values of $R$, i.e., decelerate the flow and reduce fluid velocity.

Figure 2(a-ii) demonstrates that the temperature profile increases due to rising values of $R$. In consequence, the increasing values of $R$ conduct to an increase in the boundary layer thickness. As a result the heat transfer rate reduces the present thermal buoyancy force.

\subsection{Effect of Magnetic Parameter (M)}

Figure 2(b-i) and Figure 2(b-ii) elucidates the variation of velocity and temperature profile of the flow field owing to several values of magnetic parameter $M$ whereas the other parameters are constant. Figure $2(\mathrm{~b}-\mathrm{i})$ demonstrates that for increasing values of magnetic parameters $M$, the velocity of the flow field lessens. Because when we apply a transverse magnetic field to electrically leading 
M. S. Ullah et al.
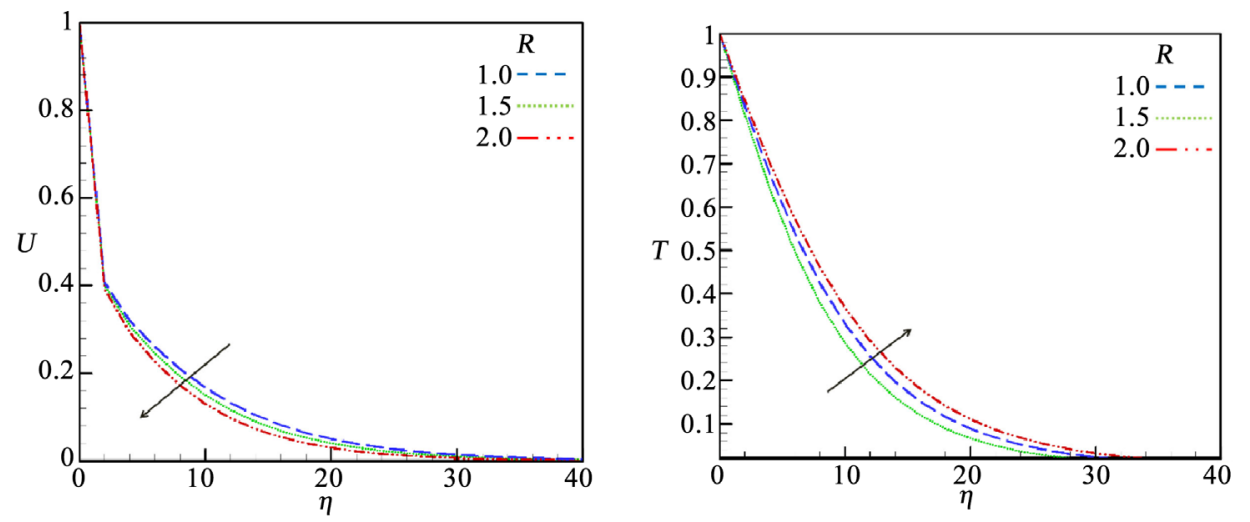

(a-i)

(a-ii)
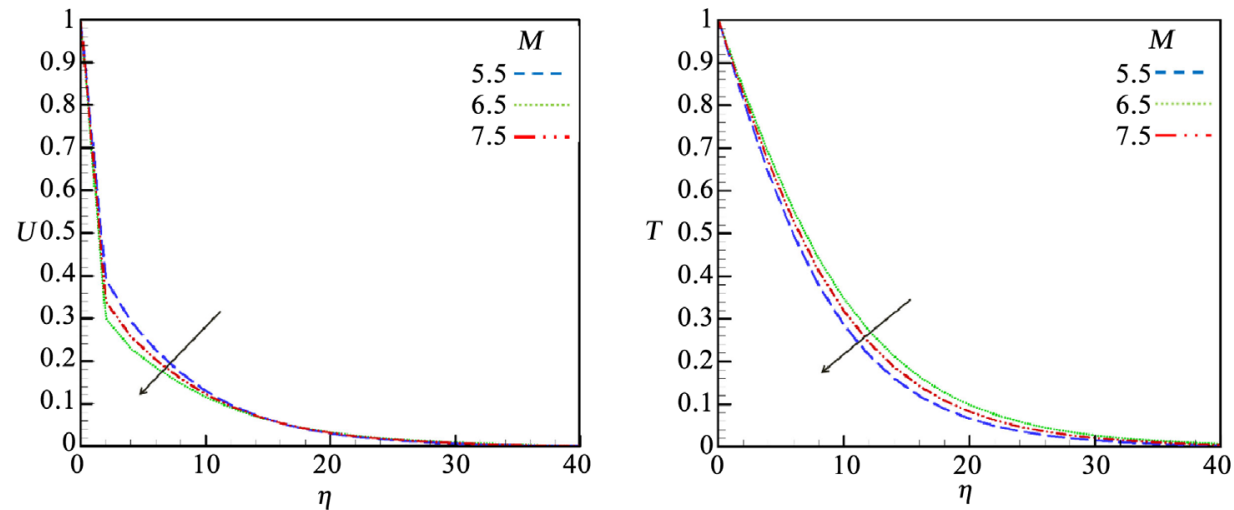

(b-i)

(b-ii)
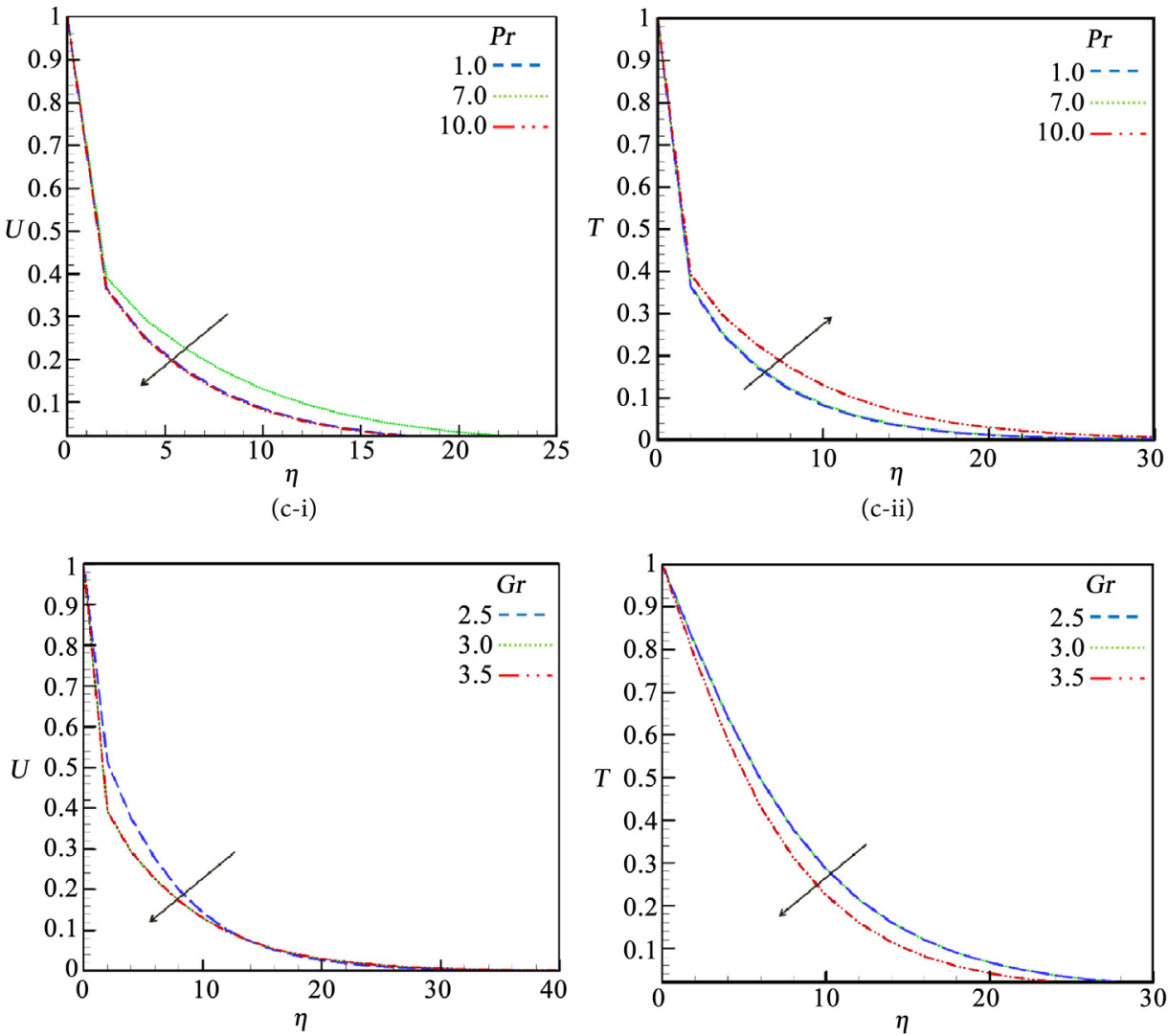

(d-i)

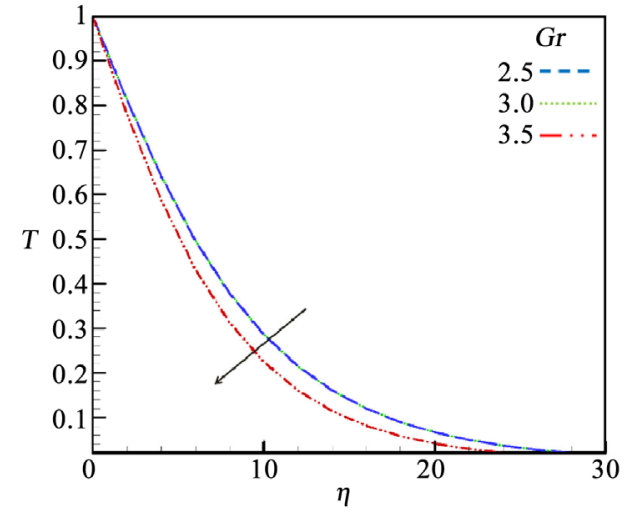

(d-ii) 

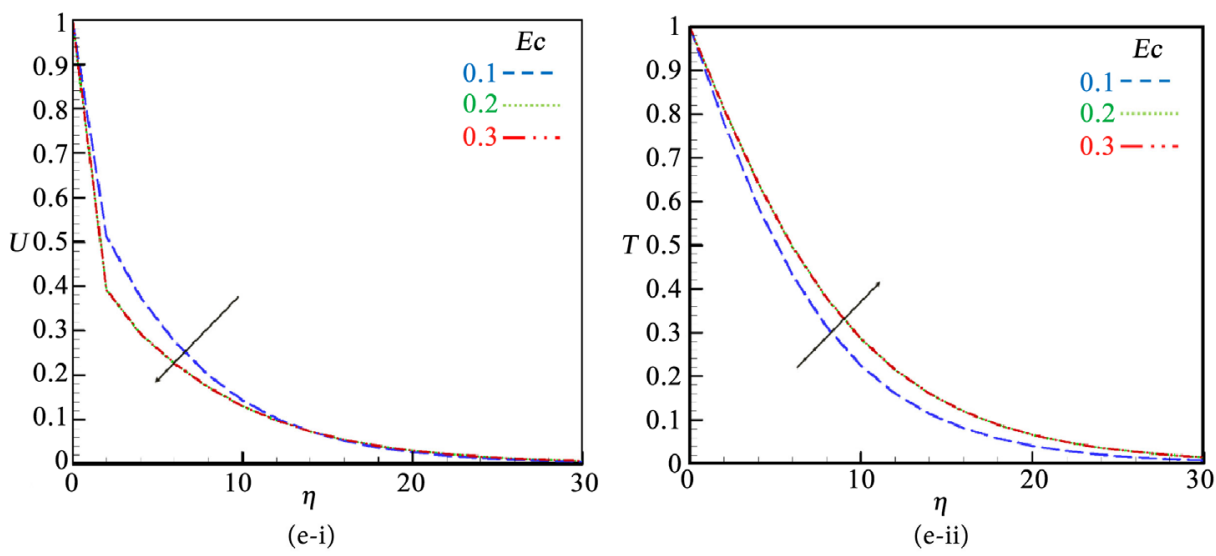

Figure 2. (a) Effect of Radiative parameter $(R)$ on velocity and temperature profile taking $G r=$ 2.5, $P r=1.0, M=5.5, E c=0.1$; (b) Effect of magnetic parameter $(M)$ on velocity and temperature profile taking $G r=2.5, R=1.0, P r=1.0, E c=0.1$; (c) Effect of Prandtl number $(P r)$ on velocity and temperature profile taking $G r=2.5, R=1.0, M=5.5, E \mathcal{C}=0.1$; (d) Effect of Grashof number $(G r)$ on velocity and temperature profile taking $R=1.0, \operatorname{Pr}=1.0, M=5.5, E \mathcal{C}=0.1$; (e) Effect of Eckert number $(E c)$ on velocity and temperature profile taking $G r=2.5, R=1.0, P r=$ $1.0, M=5.5$.

fluids, it escalates a body force prominent as Lorentz force. Furthermore, it reduces the swiftness of the movement of the fluid in the boundary layer. Figure 2(b-ii) exhibits that the temperature profiles are decreasing as a result of the ascending effect of $M$. As the temperature gradient is lessened for reducing the amount of heat convection in the flow which implies that larger values of magnetic field parameters produce lower heat transfer.

\subsection{Effect of Prandtl Number (Pr)}

Figure 2(c-i) illuminates the velocity profiles that are lessening for increasing values of Pr. Because, a higher Prandtl number creates high viscosity, consequently the velocity profiles are moving slowly. Figure 2(c-ii) displays the temperature profile which rises with the increasing of Pr. Owing to the matter, the thriving values of $\operatorname{Pr}$ are proportional to reduce thermal conductivity, that's why temperature profiles increases.

\subsection{Effect of Grashof Number (Gr)}

Figure 2(d-i) exhibits that the velocity profile decreases for rising values of $G r$. Figure 2(d-ii) elucidates that the temperature profile decrease due to thriving values of $G r$. It reveals that the growing of $G r$ decreases the rate of flow of temperature within the boundary layer.

\subsection{Effect of Eckert Number (Ec)}

Figure 2(e-i) demonstrates that the velocity profile declines because of the increasing values of $E c$. As a result, heat transfer of the flow reduced the driving force to the kinetic energy. Since scouring heating and heat energy are stored in liquid, therefore the temperature profile increases for increasing values of $E c$, 
which are illustrated in Figure 2(e-ii).

\section{Conclusions}

The governing equations for unsteady MHD free convection flow over a vertical plate have been analyzed in the entity of a magnetic field with thermal radiation. Elaborate numerical computations have been brought to pass to scrutinize the outcome of the radiative parameter $R$, magnetic parameter $M$, Prandtl number $P r$, Grashof number $G r$, and Eckert number Ec on velocity and temperature profiles. After analysis, the following conclusions are drawn:

- The offshoots of $R, M, P r, G r$, and $E c$ on velocity have shown opposite results i.e. if the values of $R, M, P r, G r$, and $E c$ increase, the velocity profiles decrease.

- The thermal boundary layer has decreased on account of the various values of $R, M$ and $G r$, whereas an opposite scenario has in $P r, E c$.

\section{Conflicts of Interest}

This manuscript has not been published and is not under consideration for publication elsewhere. We have no conflicts of interest to disclose.

\section{References}

[1] Soundalgekar, V.M., Takhar, H.S. and Vighnesam, N.V. (1960) The Combined Free and Forced Convection Flow Past a Semi-Infinite Plate with Variable Surface Temperature. Nuclear Engineering and Design, 110, 95-98. https://doi.org/10.1016/0029-5493(88)90009-X

[2] Hossain, M.A. and Takhar, H.S. (1996) Radiation Effect on Mixed Convection along a Vertical Plate with Uniform Surface Temperature. International Journal of Heat and Mass Transfer, 31, 243-248. https://doi.org/10.1007/s002310050052

[3] Das, U.N., Deka, R. and Soundalgekar, V.M. (1996) The Radiation Effects on Flow Past an Impulsively Started Infinite Vertical Plate. J. of Theo. Appl. Fluid Mech, 1, 111-115.

[4] Hossain, M.A., Alim, M.A. and Rees, D.A.S. (1999) The Effect of Radiation on Free Convection from a Porous Vertical Plate. International Journal of Heat and Mass Transfer, 42, 181-191. https://doi.org/10.1016/S0017-9310(98)00097-0

[5] Raptis, A. and Perdikis, C. (1999) Radiation and Free Convection Flow Past a Moving Plate. Applied Mechanical Engineering, 4, 817-821.

[6] Hossain, M.A. and Pop, I. (2001) Radiation Effect on Free Convection over a Vertical Flat Plate Embedded in a Porous Medium with High Porosity. International Journal of Thermal Sciences, 40, 289-295. https://doi.org/10.1016/S1290-0729(00)01210-2

[7] Chamkha, A.J. (2001) Coupled Heat and Mass Transfer by Natural Convection about a Truncated Cone in the Presence of Magnetic Field and Radiation Effects. Numerical Heat Transfer, Part A: Applications, 39, 511-530. https://doi.org/10.1080/104077801750111601

[8] Hossain, M.A., Khalil, K. and Vafai, K. (2001) Effect of Radiation on Free Convection Flow of Fluid with Variable Viscosity from a Porous Vertical Plate. International Journal of Thermal Sciences, 40, 115-124. 
https://doi.org/10.1016/S1290-0729(00)01200-X

[9] Israel-Cookey, C., Ogulu, A. and Omubo-Pepple, V.B. (2003) Influence of Viscous Dissipation and Radiation on Unsteady MHD Free-Convection Flow Past an Infinite Heated Vertical Plate in a Porous Medium with Time-Dependent Suction. International Journal of Heat and Mass Transfer, 46, 2305-2311. https://doi.org/10.1016/S0017-9310(02)00544-6

[10] Chamkha, J.A., Mujtaba, M., Quadri, A. and Issa, C. (2003) Thermal Radiation Effects on MHD Forced Convection Flow Adjacent to a Non-Isothermal Wedge in the Presence of a Heat Source or Sink. Heat and Mass Transfer, 39, 305-312. https://doi.org/10.1007/s00231-002-0353-4

[11] Abd EL-Naby, M.A., El-Barbary, E.M.E. and Abdelazem, N.Y. (2004) Finite Difference Solution of Radiation Effects on MHD Unsteady Free-Convection Flow on Vertical Porous Plate. Applied Mathematics and Computation, 151, 327-346. https://doi.org/10.1016/S0096-3003(03)00344-8

[12] Makinde, O.D. (2005) Free-Convection Flow with Thermal Radiation and Mass Transfer Past a Moving Vertical Porous Plate. International Communications in Heat and Mass Transfer, 32, 1411-1419.

https://doi.org/10.1016/j.icheatmasstransfer.2005.07.005

[13] Duwairi, H.M. (2005) Viscous and Joule Heating Effects on Forced Convection Flow from Radiate Isothermal Porous Surfaces. International Journal of Numerical Methods for Heat \& Fluid Flow, 15, 429-440. https://doi.org/10.1108/09615530510593620

[14] Damseh, R.A., Duwairi, H.M. and Al-Odat, M. (2006) Similarity Analysis of magnetic Field and Thermal Radiation Effects on Forced Convection Flow. Turkish Journal of Engineering and Environmental Sciences, 30, 83-89.

[15] Aydin, O. and Kaya, A. (2008) Radiation Effect on MHD Mixed Convection Flow about a Permeable Vertical Plate. Heat and Mass Transfer, 45, 239-246. https://doi.org/10.1007/s00231-008-0428-y

[16] Cortell, R. (2008) Effects of Viscous Dissipation and Radiation on Thermal Boundary Layer over a Nonlinearly Stretching Sheet. Physics Letters A, 372, 631-636. https://doi.org/10.1016/j.physleta.2007.08.005

[17] Deka, R.K. and Neog, B.C. (2009) Unsteady MHD Flow Past a Vertical Oscillating Plate with Thermal Radiation and Variable Mass Diffusion. Chamchuri Journal of Mathematics, 1, 79-92.

[18] Deka, R.K. and Neog, B.C. (2009) Combined Effects of Thermal Radiation and Chemical Reaction on Free Convection Flow Past a Vertical Plate in Porous Medium. Advances and Applications in Fluid Mechanics, 6, 181-195.

[19] Roy, N.C. and Hossain, M.A. (2010) The Effect of Conduction-Radiation on the Oscillating Natural Convection Boundary Layer Flow of Viscous Incompressible Fluid along a Vertical Plate. Proceedings of the Institution of Mechanical Engineers, Part C: Journal of Mechanical Engineering Science, 224, 1959-1972. https://doi.org/10.1243/09544062JMES1941

[20] Seth, G.S., Ansari, Md.S. and Nandkeolyar, R. (2011) MHD Natural Convection Flow with Radiative Heat Transfer Past an Impulsively Moving Plate with Ramped Wall Temperature. Heat and Mass Transfer, 47, 551-561. https://doi.org/10.1007/s00231-010-0740-1

[21] Das, K. (2012) Impact of Thermal Radiation on MHD Slip Flow over a Flat Plate with Variable Fluid Properties. Heat and Mass Transfer, 48, 767-778.

https://doi.org/10.1007/s00231-011-0924-3 
[22] Khan, M.S., Karim, I., Ali, L.E., et al. (2012) Unsteady MHD Free Convection Boundary-Layer Flow of a Nanofluid along a Stretching Sheet with Thermal Radiation and Viscous Dissipation Effects. International Nano Letters, 2, Article No. 24. https://doi.org/10.1186/2228-5326-2-24

[23] Rajeswara Rao, U., Ramachandra Prasad, V., Viswanath, G. and Vasu, B. (2012) Radiation Effects on Unsteady Free Convection Heat and Mass Transfer in a Walters-B Viscoelastic Flow Past an Impulsively Started Vertical Plate. International Journal of Scientific and Engineering Research, 3, 1-5.

[24] Roy, N.C., Ghosh, B.P. and Saha, L.K. (2014) Unsteady MHD Free Convection Flow along with a Vertical Plate in the Presence of Radiative Heat Flux. Applied Mathematics, 4, 77-85.

[25] Samad, M.A. and Saha, K.C. (2014) Radiative Heat and Mass Transfer of an MHD Free Convective Flow of Non-Newtonian Power-Law Fluids along a Continuously Moving Stretching Sheet with Uniform Surface Heat Flux. Dhaka University Journal of Science, 62, 37-44. https://doi.org/10.3329/dujs.v62i1.21958

[26] Fetecau, C., Vieru, D., Fetecau, C., et al. (2015) Slip Effects on the Unsteady Radiative MHD Free Convection Flow over a Moving Plate with Mass Diffusion and Heat Source. European Physical Journal Plus, 130, Article No. 6. https://doi.org/10.1140/epjp/i2015-15006-8

[27] Nagamanemma, K., Varma, S.V.K., Raju, G.S.S. and Raju, M.C. (2015) Unsteady MHD Free Convective Heat and Mass Transfer Flow near a Moving Vertical Porous Plate with Radiation \& Thermo Diffusion Effects. International Journal of Advanced Information Science and Technology (IJAIST), 38, 133-146.

[28] Tomer, V. and Kumar, M. (2015) Radiation Effects on Heat and Mass Transfer in a Steady MHD Flow over a Porous Vertical Plate. International Journal of Engineering \& Technical Research (IJETR), 3, 2454-4698.

[29] Tarammim, A., Ullah, M. and Uddin, M. (2020) A Study of Two Dimensional Unsteady MHD Free Convection Flow over a Vertical Plate. Open Journal of Fluid Dynamics, 10, 342-355. https://doi.org/10.4236/ojfd.2020.104020 